\title{
Mathematical Modelling and Computational Simulation of Mammalian Cell Cycle Progression in Batch Systems
}

\author{
Massimo Pisu, Alessandro Concas, Giacomo Cao, and Antonella Pantaleo
}

\section{ABSTRACT}

Cell cycle and its progression play a crucial role in the life of all living organisms, in tissues and organs of animals and humans, and therefore are the subject of intense study by scientists in various fields of biomedicine, bioengineering and biotechnology. Effective and predictive simulation models can offer new development opportunities in such fields. In the present paper a comprehensive mathematical model for simulating the cell cycle progression in batch systems is proposed. The model includes a structured population balance with two internal variables (i.e., cell volume and age) that properly describes cell cycle evolution through the various stages that a cell of an entire population undergoes as it grows and divides. The rate of transitions between two subsequent phases of the cell cycle are obtained by considering a detailed biochemical model which simulates the series of complex events that take place during cell growth and its division. The model capability for simulating the effect of various seeding conditions and the adding of few substances during in vitro tests, is discussed by considering specific cases of interest in tissue engineering and biomedicine.

Keywords: Cell cycle progression, cyclins and Cdks, mathematical modelling, population balance.

\author{
Published Online: January 3, 2022 \\ ISSN : 2684-5199 \\ DOI : $10.24018 / \mathrm{ejbio.2022.3.1.315}$ \\ M. Pisu* \\ Center for Advanced Studies, Research \\ and Development in Sardinia (CRS4), \\ Italy. \\ (e-mail: massimo@crs4.it) \\ A. Concas \\ Department of Mechanical, Chemical and \\ Materials Engineering, University of \\ Cagliari, Italy. \\ G. Cao* \\ Department of Mechanical, Chemical and \\ Materials Engineering, University of \\ Cagliari, Italy, and Center for Advanced \\ Studies, Research and Development in \\ Sardinia (CRS4), Italy. \\ A. Pantaleo \\ Department of Biomedical Science, \\ University of Sassari, Italy. \\ *Corresponding Author
}

\section{INTRODUCTION}

In the field of tissue engineering and biomedicine computer-aided simulation represents a powerful tool to interpret experimental trials, identify the best operating conditions to be adopted during cell cultivation, or predict/interpret the effect of specific substances on cell growth and its proliferation. Computational tools can be particularly effective and useful for the optimization of cell culture in view of the production of monoclonal antibodies (mAbs), recombinant DNA proteins and vaccines, as well as for the synthesis of ex vivo tissues/organs by means of stem cells differentiation or the expansion of specialized cells. Reliable and predictive models, on which computational tools are based to properly simulate in vitro cell culture, require an accurate description of the cell cycle that regulates the proliferative capacity of cells under normal or pathologic conditions. The typical schematization of the eukaryotic cell cycle involves four distinct phases (Murray and Hunt, 1993). A post-mitotic cell grows due to biosynthetic activities (gap phase, G1) before duplicating its DNA content (synthesis phase, S). Next, the cell enters a new gap phase (G2) wherein it grows and prepares for the subsequent division which occur during the mitotic phase (M). Under certain conditions, a cell in the stage G1 can leave the cycle reaching a specific condition of quiescence/senescence (resting phase, G0). It is well known that eucaryotic cell cycle is governed by cyclin dependent kinases (Cdks), small proteins whose activity is exerted when they associate with cyclins to form heterodimeric complexes which catalyze the transfer of phosphates from adenosine triphosphate (ATP) to specific target proteins (Morgan, 1995, 1997 and 2007; Nurse, 2000). During cell cycle, the level of active cyclin/Cdk complexes undergoes oscillations which coordinate the timing of all cellular events and drive, irreversibly, phase transitions (Nurse, 2000; Morgan, 2007). The regulatory mechanism that controls the levels of active cyclin/Cdk heterodimers, their oscillations, and consequently the progression of the cell cycle is very complex. The crucial steps involved in such a mechanism are schematically reported in Fig. 1.

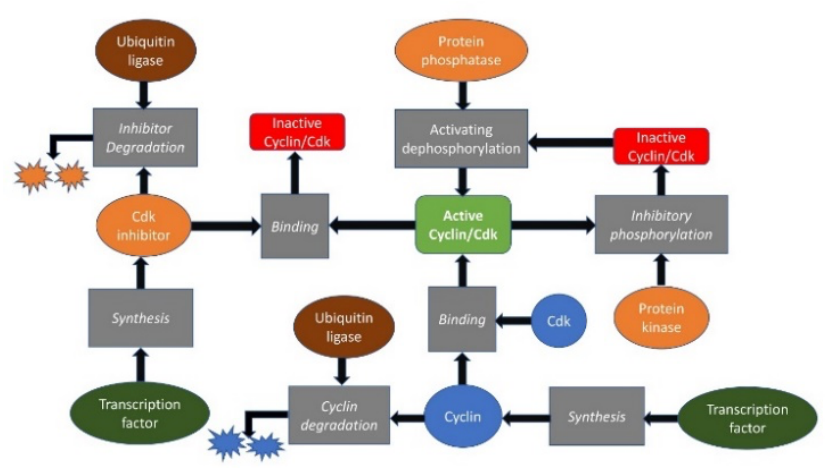

Fig. 1. Schematic representation of the main processes that regulate the level of active cyclin/Cdks complexes and their oscillations during the progress of cell cycle. 
In particular, they include the expression and degradation of cyclins, their binding with $\mathrm{Cdks}$ to form active heterodimeric complexes, then the phosphorylation or dephosphorylation processes to inactivate or activate cyclin/Cdk, and finally, the expression and degradation of inhibitory proteins which in turn may inactivate the same cyclin/Cdk heterodimers (Morgan, 2007). Since the early 90s, a significant number of mathematical models to simulate eukaryotic cell cycle progression or part of it, have been proposed in the literature. For this purpose, various approaches have been adopted, mostly based on deterministic equations even if a substantial number of models make use of stochastic, Boolean or hybrid methods (Ahmadian et al., 2020). Tyson (1991), by means of a stochastic approach, developed a mathematical model focused on the phase $\mathrm{S}$ and $\mathrm{M}$ of the cell cycle for frog eggs. Later, Chen et al. (2000 and 2004) proposed a detailed biochemistry mechanism to simulate the yeast cell cycle. Ciliberto et al. (2003), by following a deterministic approach, described the main checkpoints which underlie of the cell cycle progression for the budding yeast, while, some years later, for a generic eukaryotic cell line, Csikasz-Nagy et al. (2006) proposed a mathematical model to simulate the DNA synthesis. Next, Davidich and Bornholdt (2008) published a Boolean network model, based on the known biochemical interaction topology, to simulate cell-cycle progression for the $\mathrm{S}$. Pombe yeast. The Boolean approach does not require to define specific parameters but only the structure of the regulatory circuitry. More recently, Ahmadian et al. (2020) proposed a hybrid modelling method to simulate the cell cycle of the budding yeast by combining the deterministic approach to the stochastic one.

For the specific case of mammalian cellular line, Aguda and Tang (1999) and Qu et al. (2003) developed a detailed mathematical model to simulate the transition between G1 phase and S one. More recently, Gérard and Goldbeter (2009, 2012 and 2014) proposed a comprehensive biochemical model which describes all phases of the mammalian cell cycle. In particular, the model is capable to simulate the wellknown oscillations that cyclins and the corresponding complexes cyclin/Cdk, namely D/Ckd4-6, E/Cdk2, A/Cdk2 and $\mathrm{B} / \mathrm{Cdk} 1$, undergo during the cell cycle evolution. Weis et al. (2014) proposed an interesting data-driven approach to fit model parameters, mainly reaction rate constants, by considering various models and data reported in the literature over the mammalian cell cycle. Beside the classic optimization methods, for calibrating model parameters the authors took advantage of various techniques, among which the "simulated annealing", genetic algorithms or other stochastic methods (Weis et al., 2014). More recently, Almeida et al. (2017) developed a reduced variable mechanistic model able to capture the essential features of the mammalian cell cycle by considering a limited number of key components involved in the biological process.

In order to simulate cells growth, their replication and distribution during in vitro cultivation an effective and comprehensive model should take into account not only the detailed and complex intracellular phenomena which govern the cell life and but also the heterogeneities of the entire cell population during its evolution. Typically, cell culture is simulated by taking advantage of average cell models or by means of population balance models (PBMs) (Karra et al., 2010). With the first approach, the heterogeneities of the cell population and their distribution cannot be described. This task is well performed by PBMs which, on the contrary, cannot simulate the detailed and complex phenomena which occur inside the cell. At the same time, PBMs require the knowledge of suitable and reliable functions to describe the transition from a cell phase to the next one as well as the cell growth and its division rate (Ramkrishna, 2000). Florian and Parker (2005) developed an age-structured PBM to describe a tumor growth under chemotherapy treatment where phase transition and division rates are function of a specific critical age. Later, Liu et al. (2007) proposed a PBM with two state variables (i.e., DNA content and cell volume) to simulate the cell cycle progression observed during in vitro culture of myeloma cells. With a different approach, Sherer and Ramkrishna (2008) developed a general stochastic method for multistate systems and illustrated its advantage and potentiality in the case of cell population behavior under chemotherapy treatment. The model does not consider the complex biochemical reaction pathways which govern the cell cycle progression but describes, with a stochastic approach, the evolution of a cell population distributed in the lumped phase "G" (G0/G1), in the phase S and in the lumped one "M" (G2/M). Subsequently, Karra et al. (2010) proposed a multiscale model for the simulation of the mammalian cell culture based on kinetic equations of the main nutrients and metabolites coupled with a mass structured PBM with three subpopulation related to the lumped phases "G0/G1" and "G2/M "as well the S one. Later, Fadda et al. (2012a and 2012b) developed a multistage PBM with DNA content and cell volume as internal variables. Various functions to calculate rate of transitions from $\mathrm{G} 1$ to $\mathrm{S}$ and from $\mathrm{G} 2 / \mathrm{M}$ to G1 were used, while the transition from $\mathrm{S}$ to $\mathrm{G} 2 / \mathrm{M}$ was simulated by adopting a fully deterministic approach, based on the DNA content. More recently, Fuentes-Garì et al. (2015a) developed a multistage PBM by considering as internal variables the expression levels of cyclin E, DNA, and cyclin B, for cells in $\mathrm{G} 0 / \mathrm{G} 1, \mathrm{~S}$, and $\mathrm{G} 2 / \mathrm{M}$ phase, respectively. The same research group illustrated the capability of the proposed model in the specific case of leukemia chemotherapy treatment (Fuentes-Garì et al., 2015a). Finally, Pisu et al. (2015), on the basis of PBM approach (Fadda et al., 2012a, 2012b; Pisu et al., 2003, 2004, 2006 and 2008; Mancuso et al., 2009 and 2010), and by considering a detailed biochemical network that regulates the cell cycle of mammalian cells (Gérard and Goldbeter (2009 and 2012) proposed a novel method to link PBM to cell cycle kinetics. Specifically, the authors developed a multistage PBM involving four subpopulations (i.e., cells distributed over G0, G1, S, G2/M phases) with cell volume and dimensionless age of a cell in each phase as internal variables. The residence time of a cell in a given phase, appearing in PBM parameters, was calculated by considering a specific biochemical reactions model. Such an approach neglects the biochemical pathways involved in some important extracellular signaling which may affect the cell cycle duration as, for example, the regulatory mechanisms due to the presence external extracellular matrix (ECM) and growth factors.

In this work a novel mathematical model for simulating the cell cycle progression in batch systems (Petri dish) is 
proposed. The model is based on a multistage population balance with two internal variables (i.e., cell volume and a specific dimensionless age) for the simulation of cell cycle progression through the different phases experienced by each cell of the entire population. The rate of transitions between two subsequent phases of the cell cycle are obtained by means of a detailed biochemical model which simulates the series of complex events that take place during the cell growth and its division. With respect to the work by Pisu et al. (2015), a more detailed kinetic network, which includes the regulatory action due to the extracellular signaling induced by external GF and ECM and their direct interaction with the cells cycle, is taken into account. The potential of the mathematical model has been tested by considering some specific procedures taking place during in vitro trials and in particular, the effect of seeding conditions and the addition of suitable substances in the culture medium.

\section{MAthematicAl Model}

The mathematical model proposed in this work is divided in two modules. The first one takes advantage of a comprehensive model for the description of the complex network of biochemical reactions that governs the progression of a single cell cycle, while the second one is based on a PBM where the cell distribution in each cell cycle phase is defined in terms of size (cell volume) and a proper maturation age (dimensionless) for transition.

The kinetic module was developed on the basis of the mathematical model proposed by Gérard and Goldbeter (2014) which is schematically reported in Fig. 2.

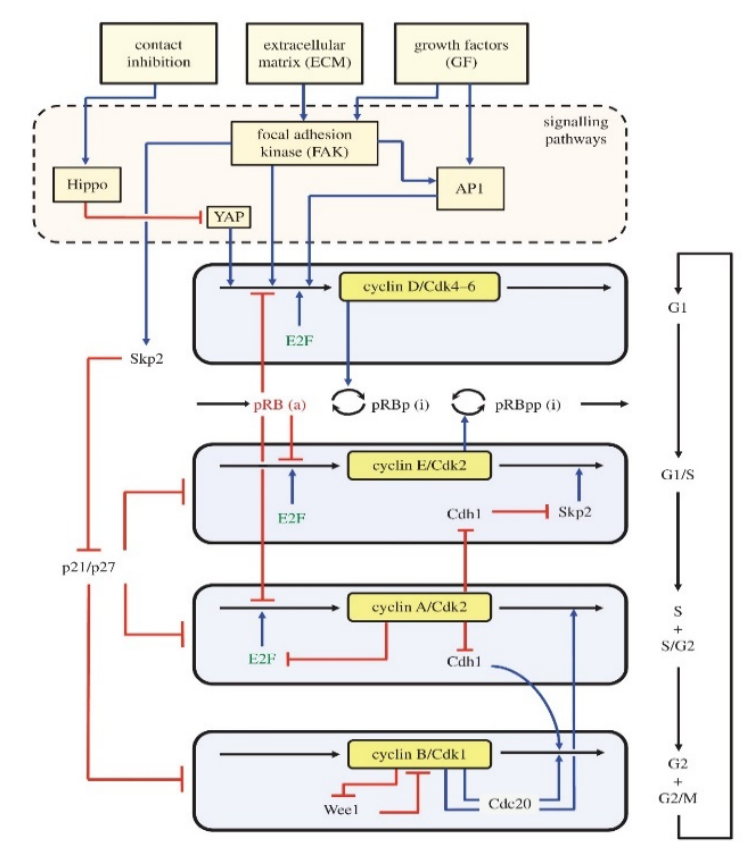

Fig. 2. Biochemical model for the cyclin/Cdk network driving the mammalian cell cycle (taken from Gérard and Goldbeter, 2014).

The intracellular reaction network is divided into four sections each one focused on a specific cyclin/Cdk complex (i.e., D/Ckd4-6, E/Cdk2, $\mathrm{A} / \mathrm{Cdk} 2$ and $\mathrm{B} / \mathrm{Cdk} 1$ ). The heterodimer D/Cdk4-6, after its activation, starts the cell cycle and promotes the progression in $\mathrm{G} 1$ phase, the complex $\mathrm{E} / \mathrm{Cdk} 2$ elicits the $\mathrm{G} 1 \rightarrow \mathrm{S}$ transition, while the complex
$\mathrm{A} / \mathrm{Cdk} 2$ sustains the progression in $\mathrm{S}$ and the $\mathrm{S} \rightarrow \mathrm{G} 2$ conversion. Finally, the complex B/Cdk1 triggers the $\mathrm{G} 2 \rightarrow \mathrm{M}$ transition which leads to the cell division. The biochemical model also describes the regulatory effect of some proteins (Cdh1, Cdc20, Wee1, Skp2 and cdc25 phosphatase) and Cdks inhibitors (p21/p27), the synthesis and degradation of cyclins, their binding with Cdks, and the opposed effect of the tumor suppressor $\mathrm{pRB}$ and the transcription factor E2F. The extended model by Gérard and Goldbeter (2014), compared to the one previously developed by the same authors (Gérard and Goldbeter, 2009 and 2012) includes the effect of some extracellular signaling and in particular the crucial influence of an external growth factor GF. The latter one elicits the activation of signaling pathways leading to the synthesis of the transcription factor AP1 which in turn, together with E2F, promotes the synthesis of cyclin D starting the cell cycle (Fig. 2 ). The effect of an extracellular signaling induced by GFs or, similarly, by other species affecting the cell cycle, can be accounted for by taking advantage of the following equation which describes the expression level of the transcription factor AP1 factor (Gérard and Goldbeter, 2014):

$$
\begin{aligned}
& \frac{d[A P 1]}{d t}=\left(v_{S A P 1} \cdot\left(\frac{[G F]}{K_{A G F}+[G F]}\right)+v_{S A P 2} \cdot\left(\frac{[F A K]}{K_{A F A K}+[F A K]}\right)-k_{D A P 1} .\right. \\
& [A P 1]) \cdot \varepsilon
\end{aligned}
$$

where $v_{S A P 1}$ is the rate of synthesis of the transcription factor AP1 depending on GF, $K_{A G F}$ is the Michaelis constant for synthesis of AP1 induced by GF, FAK represents expression level of the focal adhesion kinase, $v_{S A P 2}$ is the rate of synthesis of the transcription factor AP1 depending on FAK, $K_{A G F}$ is the Michaelis constant for synthesis of AP1 induced by FAK, finally $\varepsilon$ represents a scale factor (Gérard and Goldbeter, 2014) . The biochemical model also accounts for the extracellular signaling induced by contact inhibition which may affect the cell proliferation at high cell density, and the presence of ECM which in turn can affect the cell cycle progression through FAK1 (cf. Fig. 2). Overall, the model proposed by Gérard and Goldbeter (2014), considered in the present work, simulates a biochemical network of 42 reacting species (namely, growth and transcription factors, cyclins, Cdks, complexes cyclin/Cdk in active or inactive form, phosphorylated or unphosphorylated proteins, protein phosphatases, Cdks inhibitors). The resulting system of 42 ordinary differential equations (ODEs), which properly describes the dynamics and the typical oscillations of mammalian cell cycle, involves over two hundred parameters. For the sake of brevity all model equations and related parameters are not reported in this paper, thus for all details the interest reader should refer to the original work by Gérard and Goldbeter (2014).

In order to simulate the progression in $\mathrm{S}$ phase and the transition $S \rightarrow G 2$, it is necessary to describe also the DNA replication kinetics neglected in the original work by Gérard and Goldbeter (2014). As schematically illustrated in Fig. 3, at the beginning of the synthesis phase, $\mathrm{S}$, the complex $\mathrm{E} / \mathrm{Cdk} 2$ activates the anchoring factor $\mathrm{Cdc} 45$, which permits DNA polymerase $\alpha$ to start the replication. The kinase ATR is activated by binding the RNA primer synthesized by DNA polymerase $\alpha$. Next, ATR promotes the activation of the kinase Chk1 which, in turn, inhibits the Cdc25 phosphatases, thus preventing the activation of the complexes E/Cdk2, $\mathrm{A} / \mathrm{Cdk} 2$ and $\mathrm{E} / \mathrm{Cdk} 1$ during DNA replication (Gérard and 
Goldbeter, 2009 and 2012). In the light of the considerations above, it is possible to incorporate in the model five additional equations, i.e., eqs (40)-(44) reported by Gérard and Goldbeter (2009), which describe the temporal behavior of Cdc45, DNA polymerase $\alpha$, RNA primer, ATR and Chk1, respectively. The resulting system of 47 ordinary differential equations (ODEs), which allows one to calculate the duration of each one cell cycle phase, is solved through standard numerical libraries based on Gear method (IMSL).

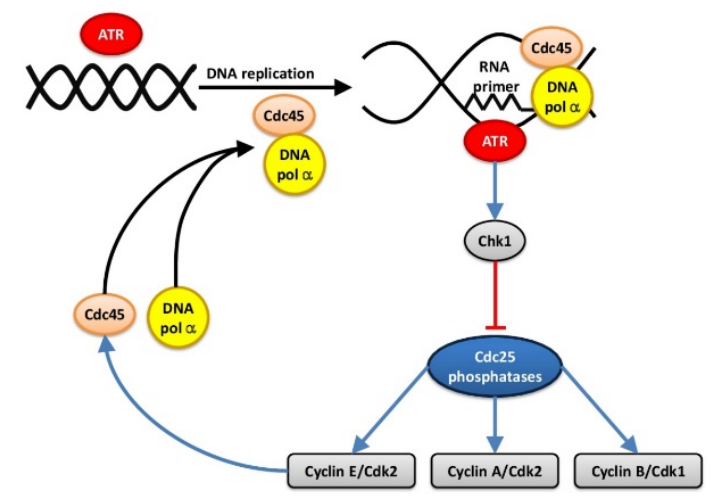

Fig. 3. The reaction network for DNA replication (taken from Gérard and Goldbeter, 2009 and 2012).

The second part of the model is based on a multi-staged population balance with two internal variables, namely the cell volume, $v$, and the dimensionless phase duration, $\xi$, which ranges between 0 (newborn cell) and 1 (cell entering the next phase). By considering a monolayer of cells evenly distributed on the surface of the cultivation system (Petri dish), lumping G2 and M phase in the single one (G2M) and assuming negligible cell death terms as well as the transition $\mathrm{G} 1 \rightarrow \mathrm{G} 0$ irreversible, the population balance equations can be written as

$$
\begin{aligned}
& \frac{\partial n^{G 0}(v, t)}{\partial t}=[1-\Phi(\mathrm{t})] \Gamma_{v}^{G 1}(v) \int_{0}^{1} n^{G 1}(v, \xi, t) d \xi \\
& \frac{\partial n^{G 1}(v, \xi, t)}{\partial t}+\frac{\partial\left[r_{v}^{G 1}(v) n^{G 1}(v, \xi, t)\right]}{\partial v}+\frac{\partial\left[r_{\xi}^{G 1} n^{G 1}(v, \xi, t)\right]}{\partial \xi}= \\
& =-[1-\Phi(\mathrm{t})] n^{G 1}(v, \xi, t) \Gamma_{v}^{G 1}(v) \\
& \frac{\partial n^{S}(v, \xi, t)}{\partial t}+\frac{\partial\left[r_{v}^{S}(v) n^{S}(v, \xi, t)\right]}{\partial v}+\frac{\partial\left[r_{\xi}^{S} n^{S}(v, \xi, t)\right]}{\partial \xi}=0 \\
& \frac{\partial n^{G 2 M}(v, \xi, t)}{\partial t}+\frac{\partial\left[r_{v}^{G 2 M} n^{G 2 / M}(v, \xi, t)\right]}{\partial v}+\frac{\partial\left[r_{\xi}^{G 2 M} n^{G 2 M}(v, \xi, t)\right]}{\partial \xi}=0
\end{aligned}
$$

along with the initial (ICs) and boundary conditions (BCs)

$n^{G 0}(v, t)=n_{0}^{G 0}(v)$ for $t=0$ and $\forall v$

$n^{P}(v, \xi, t)=n_{0}^{P}(v, \xi)$ for $t=0$ and $\forall v, \xi$ with $P=G 1, S, G 2 M$

$$
r_{\xi}^{G 1} n^{G 1}(v, \xi, t)=2 \int_{v_{\min }}^{v_{\max }} r_{\xi}^{G 2 M} n^{G 2 M}(v, 1, t) p\left(v, v^{\prime}\right) d v^{\prime}
$$$$
\text { for } \xi=0 \text { and } \forall v, t>0
$$

$$
\begin{array}{ll}
n^{G 0}(v, t)=0 & \text { for } v=v_{\text {min }} \text { and } t>0 \\
n^{P}(v, \xi, t)=0 & \text { for } v=v_{\text {min }} \text { and } \forall \xi, t>0
\end{array}
$$
with $P=G 1, S, G 2 M$

$r_{\xi}^{S} n^{S}(v, \xi, t)=r_{\xi}^{G 1} n^{G 1}(v, 1, t)$ for $\xi=0$ and $\forall v, t>0$

$$
r_{\xi}^{G 2 M} n^{G 2 M}(v, \xi, t)=r_{\xi}^{S} n^{S}(v, 1, t) \text { for } \xi=0 \text { and } \forall v, t>0
$$

where $n^{G 0}(v, t)$ represents the monodimensional number density distribution of cells in G0 phase, $n^{P}(v, \xi, t)$ is the bidimensional number density distribution of cells in the generic phase $\mathrm{P}=\mathrm{G} 1, \mathrm{~S}, \mathrm{G} 2 \mathrm{M}, \quad r_{v}^{\mathrm{P}}(v)$ and $r_{\xi}^{P}$ with $\mathrm{P}=\mathrm{G} 1, \mathrm{~S}, \mathrm{G} 2 \mathrm{M}$, are the time rates of change of the variables $v$ and $\xi$, respectively, $\Gamma_{v}^{G 1}(v)$ appearing in (2) and 3$)$ is the rate of transition from phase G1 to G0 one, while $\Phi(\mathrm{t})$ is the geometric limiting factor for simulating the well-known "cell contact inhibition" which may become significant at high level of the cell density (Fadda et al., 2012a). Beside the accumulation term, the left-hand-side of (2) for G0 phase includes an advection term on the internal variable $v$, while in Eqs (3), (4) and (5) two advection terms are considered to account for the growth on volume $v$ and dimensionless age $\xi$ of cells in the phase G1, S and G2M, respectively. The righthand-side of (2) and (3) accounts for the cells that leave, irreversibly, the phase G1 entering the G0 resting phase. Finally, in Equations (3), (4) and (5), the amount of cells which leave G1, S and G2M phase, respectively, is intrinsically taken into account by the advection term on the internal variable $\xi$ when its value attains the upper limit (i.e., $\xi=1)$. The rate of the irreversible transition $\mathrm{G} 1 \rightarrow \mathrm{G} 0$, $\Gamma_{v}^{G 1}(v)$, can be expressed as follows (Fadda et al., 2012a; Pisu et al., 2015):

$\Gamma_{v}^{G 1}(v)=r_{v}^{G 1}(v) \frac{f(v)}{1-\int_{0}^{v} f\left(v^{\prime}\right) d v^{\prime}}$

where $f(v)$ is the probability density function of transition of a cell with volume $v$ characterized by two adjustable parameters, namely w and $\lambda$ (Hatzis et al., 1995):

$f(v)=\frac{w}{\lambda w} v^{w-1}$

The function $p\left(v, v^{\prime}\right)$, appearing in (8), represents an unequal partitioning distribution, since after the division of a mother cell the two daughter cells may have a different volume (Hatzis et al., 1995):

$p\left(v, v^{\prime}\right)=\frac{1}{\beta(q, q)} \frac{1}{v^{\prime}}\left(\frac{v}{v^{\prime}}\right)^{q-1}\left(1-\frac{v}{v^{\prime}}\right)^{q-1}$

where $\beta(q, q)$ is the symmetrical beta function on the parameter $\mathrm{q}$. The volume growth rates, $r_{v}^{\mathrm{P}}(v)$, in (3)-(6), are assumed linear (Fadda et al., 2012a), thus satisfying the containment conditions (i.e., $r_{v}^{P}(0)=0$ ) (Fredrickson and Mantzaris, 2002):

$r_{v}^{P}(v)=\frac{d v}{d t}=k_{v} v$ with $P=G 1, S, G 2 M$ 
It is worth noting that, BCs (9) and (10) satisfy the regularity condition when $v_{\text {min }}>0$ (Fadda et al., 2012a; Fredrickson and Mantzaris, 2002).

The time rate of change of internal variable $\xi$ can be expressed as:

$r_{\xi}^{P}=\frac{d \xi}{d t}=\frac{1}{\tau^{P}}$ with $P=G 1, S, G 2 M$

where $\tau^{P}$ is the duration of the generic phase $\mathrm{P}$ computed by taking advantage of the kinetic module. On the basis of the equation (17), the advection terms on the variable $\xi$, appearing in (3), (4) an (5), assume the classical form they have in age structured population balances (Ramkrishna, 2000). The geometric limiting factor, $\Phi(\mathrm{t})$, appearing in equations (2) and (3), accounts for the cell contact inhibition which leads the transition from the phase G1 to the resting one G0. For anchorage-dependent cells the value of $\Phi(t)$, depending on the level of cell density attained during cultivation in a Petri dish having area $A_{P}$, ranges from 1 (no limitation) to 0 (confluence, no space available for cell growth). For the complete description of the term $\Phi(t)$, which is modelled by considering only one parameter (i.e., the interstitial constant $\alpha$ ), the interested reader should refer to the original work by Fadda et al. (2012a). Along similar lines, the term $\Phi(\mathrm{t})$ can be defined for any cultivation batch system (well plates, flasks).

Finally, from the number density distribution, the cell count of each phases can be obtained as follows:

$$
\begin{aligned}
& N^{G 0}(t)=\int_{v_{\text {min }}}^{v_{\text {max }}} n^{G 0}(v, t) d v \\
& N^{P}(t)=\int_{v_{\text {min }}}^{v_{\text {max }}} \int_{0}^{1} n^{P}(v, \xi, t) d v d \xi \text { with } P=G 1, S, G 2 M
\end{aligned}
$$

and the total cell count $N(t)$ can be calculated as:

$$
N(t)=N^{G 0}(t)+N^{G 1}(t)+N^{S}(t)+N^{G 2 M}(t)
$$

Equations (2)-(5) represent a system of partial differential equations (PDEs) in the independent variables $\mathrm{t}, v \xi$, along with the ICs and BCs (6)-(12). In order to numerically solve such a system, we implemented the method of lines, thus the domain of the variables $v$ and $\xi$ was suitably divided by using constant step size meshes (100 and 300 grid points, respectively) and the partial derivatives, with respect to the same variables, were discretized by backward finite differences. The resulting system of ordinary differential equations (ODEs) in the time variable was integrated as an initial value problem by taking advantage of standard numerical libraries (Gear method, IMSL). Computational run of code, written in fortran language, were performed on nodes of PC clusters (HP, Dual CPU Quadcore at $2.8 \mathrm{GHz}$, operating system Centos7.0).

\section{RESUlts AND Discussion}

The model, described in the previous section, is capable of simulating, for various operating conditions, the cultivation of mammalian cells performed in batch systems, as for example Petri dishes, where monolayer adherent cells may grow and proliferate in the presence of specific culture medium. The replication time and consequently the evolution of the cell culture depend mainly on the cell type and its intrinsic metabolism which in turn can be affected by external chemical or mechanical stimuli. Firstly, let us consider as base case a cell cycle with a circadian rhythm (i.e., $24 \mathrm{~h}$ of cell cycle duration). This situation can be properly simulated by the kinetic module as shown in Fig. 4, where the expression levels of the active complexes D/Ckd4-6, E/Cdk2, $\mathrm{A} / \mathrm{Cdk} 2$ and $\mathrm{B} / \mathrm{Cdk} 1$ as well as the DNA polymerase $\alpha$ (i.e, DNA Pol. $\alpha$ ) content are reported as a function of time.

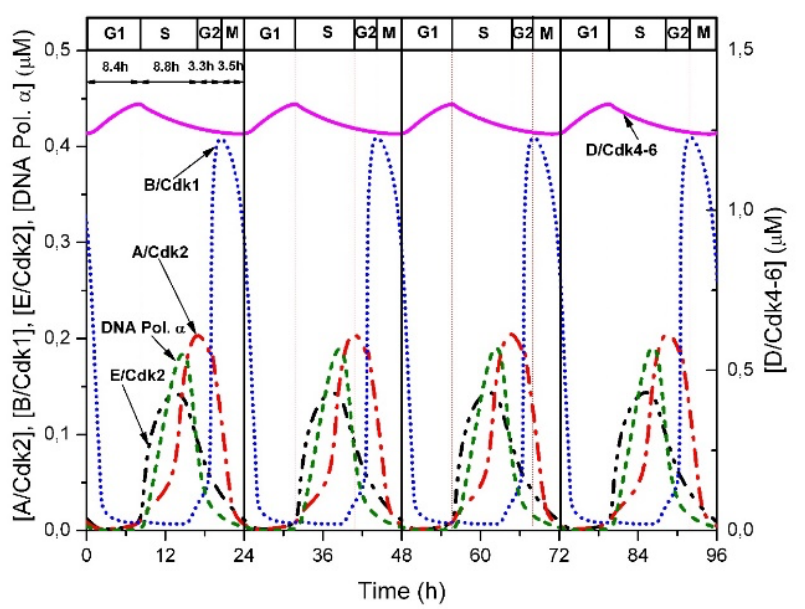

Fig. 4. Expression levels of the main Cyclin-Cdk complexes and of the DNA polymerase $\alpha$ content as a function of time. The duration of cell cycle is $24 \mathrm{~h}$.

In this simulation, as far as the biochemical model schematically illustrated in Fig. 2, we took advantage of all data and parameters reported by Gérard and Goldbeter (2014), while for the DNA replication submodule, schematically illustrated in Fig. 3, all data and parameters were taken from Gerard and Goldbeter (2009) except for the scaling factor, $\varepsilon$. In the present work, the latter one, was set equal to 14 in order to ensure a cell cycle lasting 24 hours. For the sake of brevity data (initial concentration of all 47 involved biochemical species) and model parameters (over two hundred) are not reported in this paper, thus, for all details, the interested reader should refer to the original works (Gérard and Goldbeter, 2009, 2012 and 2014). From Fig. 4 it is possible to observe the typical oscillation faced by the active complexes D/Ckd4-6, E/Cdk2, A/Cdk2 and B/Cdk1 during the cell cycle which is replicated each 24 hours. The duration of a single phase $\tau^{P}$, with $\mathrm{P}=\mathrm{G} 1, \mathrm{~S}, \mathrm{G} 2, \mathrm{M}$, can be computed on the basis of such oscillating behavior. After the activation of the complex D/Ckd4-6 the cell cycle starts in G1 phase. The heterodimer $\mathrm{D} / \mathrm{Ckd} 4-6$ sustains the cell progression in G1 and when its expression level attains the peak the transition $\mathrm{G} 1 \rightarrow \mathrm{S}$ takes place $\left(\mathrm{t}=\tau^{G 1}=8.4 \mathrm{~h}\right)$. Contextually, the complex E/Cdk2 and consequently the DNA polymerase $\alpha$ rapidly increase to ensure the DNA replication in $\mathrm{S}$ phase. When the levels of $\mathrm{E} / \mathrm{Cdk} 2$ and the DNA polymerase $\alpha$ diminish and the complex $\mathrm{A} / \mathrm{Cdk} 2$ displays its peak, the transition to the next phase $\mathrm{G} 2$ occurs ( $\mathrm{t}$ $=17.2 \mathrm{~h} ; \tau^{S}=8.8 \mathrm{~h}$ ). During the gap phase $\mathrm{G} 2$ the expression level of $\mathrm{B} / \mathrm{Cdk} 1$ increases to sustain the cell progression until its expression level attains the peak, thus eliciting the transition $\mathrm{G} 2 \rightarrow \mathrm{M}\left(\mathrm{t}=20.5 \mathrm{~h} ; \tau^{G 2}=3.3 \mathrm{~h}\right)$. 
Finally, at the end of the mitotic process, the expression levels of $\mathrm{E} / \mathrm{Cdk} 2$ and $\mathrm{A} / \mathrm{Cdk} 2$ decrease and approach zero, while the level of D/Ckd4-6 attains its minimum value before starting to grow again at the beginning of the new G1 phase $\left(\mathrm{t}=24 \mathrm{~h} ; \tau^{M}=3.5 \mathrm{~h}\right)$. Overall, the lumped phase G2M, considered in PBM, displays a duration of $\tau^{G 2 M}=\tau^{G 2}+$ $\tau^{M}=6.8 \mathrm{~h}$. It is important to note that, the level of cyclin D/Cdk4-6, in the Fig. 4 represented by the sum of its free form and the bound one to $\mathrm{p} 21 / \mathrm{p} 27$, remains elevated during the cycle, accordingly with experimental observations (Gérard and Goldbeter, 2009; van Vugt et al., 2004). The phase duration time, computed by taking advantage of the biochemical kinetic module, permits to estimate the rate $r_{\xi}^{P}=\frac{1}{\tau^{P}}$, with $\mathrm{P}=\mathrm{G} 1, \mathrm{~S}, \mathrm{G} 2 \mathrm{M}$, required by the $\mathrm{PBM}$, thus, for this base case, $\quad r_{\xi}^{G 1}=\frac{1}{\tau^{G 1}}=0.1190=\mathrm{h}^{-1}, \quad r_{\xi}^{S}=\frac{1}{\tau^{S}}=$ $0.1136=\mathrm{h}^{-1}, r_{\xi}^{G 1}=\frac{1}{\tau^{G 2 M}}=0.1470=\mathrm{h}^{-1}$.

Let us now consider some effects on in vitro cultivation due to different operating conditions, as for example when changing the initial seeding in terms of cell number and their distribution over the phase G1, S, G2M. For a first analysis let us assume an initial distribution with cells belonging only to $\mathrm{G} 1$ phase $\left(\right.$ i.e., $n_{0}^{G 0}(v)=n_{0}^{S}(v, \xi)=n_{0}^{G 2 M}(v, \xi)=0$ ), expressed with the following bivariate normal gaussian distribution on the variables $v$ and $\xi$ :

$n_{0}^{G 1}(v, \xi)=\frac{N_{0}}{2 \pi \sigma_{v} \sigma_{\xi}} \exp \left(-\frac{1}{2}\left[\frac{\left(v-\mu_{v}\right)^{2}}{\sigma_{v}^{2}}+\frac{\left(\xi-\mu_{\xi}\right)^{2}}{\sigma_{\xi}^{2}}\right]\right)$

where $N_{0}$ is initial the total cell number, $\mu_{v}$ and $\mu_{\xi}$ are the mean values of the variables $v$ and $\xi$, respectively, and $\sigma_{v}$ and $\sigma_{\xi}$ their corresponding standard deviations. Such a distribution with cells belonging only to G1 phase is used to define the initial conditions of (6) and (7). Simulation results, obtained by taking advantage of the PBM computational module, are reported in Fig. 5.

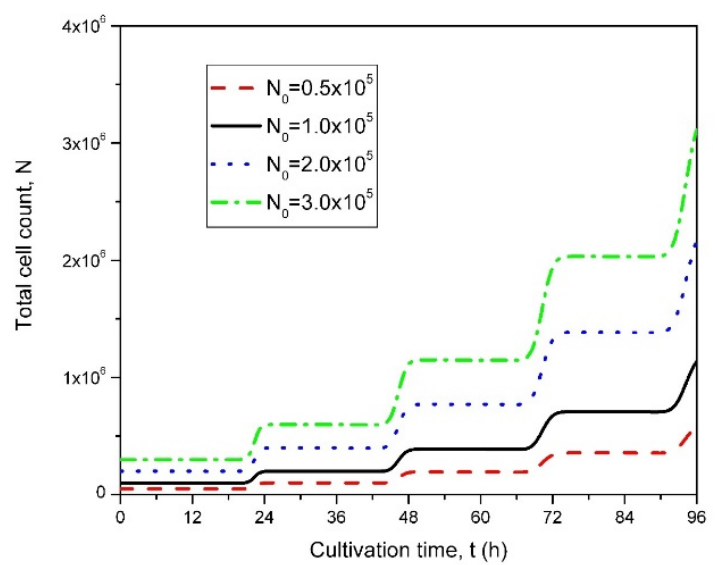

Fig. 5. Total cell count as a function of time at various initial seeding conditions (i.e., $N_{0}=0.5 \times 10^{5} ; 1.0 \times 10^{5} ; 2.0 \times 10^{5} ; 3.0 \times 10^{5}$ ).

In particular, results shown in Fig. 5, are represented in terms of total cell number as a function of the cultivation time and for various seeding conditions (i.e., $N_{0}=0.5 \times 10^{5}$; $\left.1.0 \times 10^{5} ; 2.0 \times 10^{5} ; 3.0 \times 10^{5}\right)$. All model parameters used in this simulation are reported in Table I.
TABLE I: MODEL PARAMETERS

\begin{tabular}{cccc}
\hline \hline Par. & Value & Unit & Reference \\
\hline \hline$N_{0}$ & $0.5 \times 10^{4} \div 3.0 \times 10^{5}$ & Cells & This work \\
$\mu_{v}$ & $1.5 \times 10^{3}$ & $\mu \mathrm{m}^{3}$ & This work \\
$\sigma_{v}$ & $4.0 \times 10^{2}$ & $\mu \mathrm{m}^{3}$ & This work \\
$\mu_{\xi}$ & 0.2 or 0.5 & $\mathrm{~h}^{-1}$ & Pisu et al. $(2015)$ \\
$\sigma_{\xi}$ & 0.01 or 0.15 & - & Pisu et al. $(2015)$ \\
$r_{\xi}^{G 1}$ & 0.1190 or 0.1587 & $\mathrm{~h}^{-1}$ & This work \\
$r_{\xi}^{S}$ & 0.1146 or 0.1887 & $\mathrm{~h}^{-1}$ & This work \\
$r_{\xi}^{G 2 M}$ & 0.1471 or 0.1190 & $\mathrm{~h}^{-1}$ & This work \\
$A_{P}$ & $10 \times 10^{8}$ & $\mu \mathrm{m}^{2}$ & Pisu et al. $(2015)$ \\
$\alpha$ & 1.6 & - & Fadda et al. $(2012 \mathrm{~b})$ \\
$k_{v}$ & 0.035 & $\mathrm{~h}^{-1}$ & Fadda et al. $(2012 \mathrm{~b})$ \\
$v_{\min }$ & 20.0 & $\mu \mathrm{m}^{3}$ & Fadda et al. $(2012 \mathrm{~b})$ \\
$v_{\max }$ & 7000.0 & $\mu \mathrm{m}^{3}$ & Fadda et al. $(2012 \mathrm{~b})$ \\
$w$ & 7.8 & - & Fadda et al. $(2012 \mathrm{~b})$ \\
$\lambda$ & $1.78 \times 10^{3}$ & $\mu \mathrm{m}^{3}$ & Fadda et al. $(2012 \mathrm{~b})$ \\
$q$ & 40 & - & Mancuso et al. $(2009)$ \\
\hline \hline
\end{tabular}

Specifically, as far as the bivariate normal gaussian distribution, the mean values and the corresponding standard deviation of the variable $\xi$, are set to $\mu_{\xi}=0.2$ and $\sigma_{\xi}=0.01$, respectively, while the time rates of change on the same variable $\xi$, are $r_{\xi}^{G 1}=0.1190 \mathrm{~h}^{-1}, r_{\xi}^{S}=0.1136 \mathrm{~h}^{-1}, r_{\xi}^{G 2 M}=$ $0.1470 \mathrm{~h}^{1}$. By observing Fig. 5, as it might be expected, the increase of $\mathrm{N}_{0}$ leads to an augmentation of the cell number at any cultivation time. It is also apparent that each growth curve displays the typical behavior with stationary periods and subsequent incremental steps due to the time needed to complete the cell cycle whose duration is $24 \mathrm{~h}$, consistently to what calculated by the kinetic module (cf. Fig. 4). Moreover, from Fig. 5, it can be observed the typical lag time due to acclimation period experienced by cells at the start of cultivation. In fact, the number of cells may increase only by undergoing the transition $\mathrm{G} 2 \mathrm{M} \rightarrow \mathrm{G} 1$ which requires a period of time since cells are initially synchronized only in G1 phase, as imposed by the initial conditions for this simulation. By observing Fig. 5, the initial lag time for the cell expansion is about $22 \mathrm{~h}$, according to the time required by the cells, initially synchronized in G1-phase, to complete the cell cycle, having them an initial mean value age of $\mu_{\xi} \cdot \tau^{G 1}=0.2$. $8.4=1.68 \mathrm{~h}$.

Let us now analyze the behavior of a cell population during in vitro cultivation by focusing on each single phase of the cell cycle. In Fig. 6 model results in terms of cell number distributed in G0, G1, S and G2M phases and total cell count are shown as a function of cultivation time for two specific initial seeding condition (i.e., $\mathrm{N}_{0}=1.0 \times 10^{5}$ and $3.0 \times 10^{5}$, $6 \mathrm{a}$ and $6 \mathrm{~b}$, respectively). As it can be observed in Fig. 6, cell count evolution in G1, S and G2M shows a well-known behavior oscillating between a minimum value to a maximum one. Cultivation starts with cells synchronized in the phase G1 as imposed by the initial conditions, therefore cells belonging to $\mathrm{S}$ stage can appear only when the maturation in the phase G1 is completed. The increase of cell number in the $S$ phase corresponds to a reduction of cells number in the G1 one at the occurrence of transition $\mathrm{G} 1 \rightarrow \mathrm{S}$. Similarly, the same behavior can be observed for the other transitions $\mathrm{S} \rightarrow \mathrm{G} 2 \mathrm{M}$ and $\mathrm{G} 2 \mathrm{M} \rightarrow \mathrm{G} 1$. From Fig. 6 it is also apparent that such phase transitions occur coherently with the maturation age for transition calculated by the biochemical kinetic module (i.e., $\tau^{G 1}=8.4 \mathrm{~h} ; \tau^{S}=8.8 \mathrm{~h} ; \tau^{G 2 M}=6.8 \mathrm{~h}$ ). It is important to note that, the time required for the first transition $\mathrm{G} 1 \rightarrow \mathrm{S}$ is 
lower than $\tau^{G 1}$ since the initial age of cells (mean value $\mu_{\xi} \cdot \tau^{G 1}=1.68 \mathrm{~h}$ ), as imposed in the initial conditions, is greater than 0 .
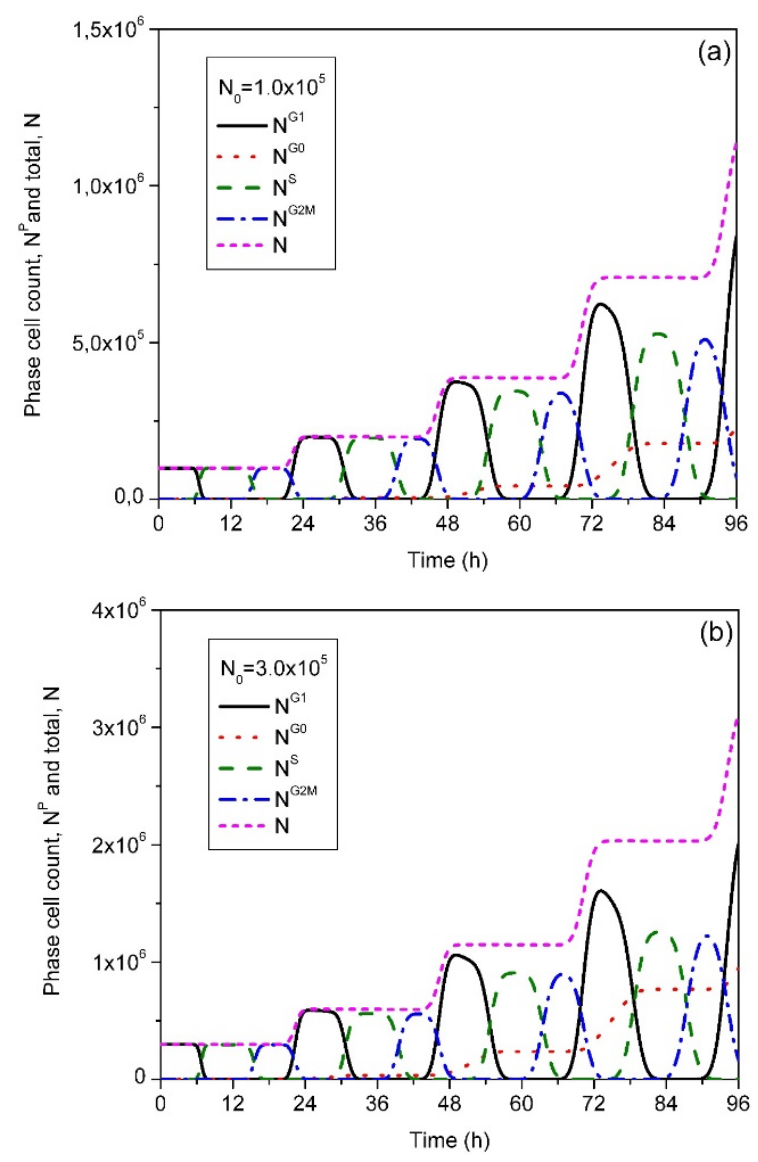

Fig. 6. Phase cell count, NP with $\mathrm{P}=\mathrm{G} 1, \mathrm{G} 0, \mathrm{~S}, \mathrm{G} 2 \mathrm{M}$, and total one, $\mathrm{N}$, as a function of time at different initial seeding condition: (a) $\mathrm{N}_{0}=1.0 \times 10^{5}$; (b) $\mathrm{N}_{0}=3.0 \times 10^{5}$.

As far as the resting phase G0, by observing Fig. 6, the cell number evolution during the cultivation displays an oscillating "step" behavior. In fact, cell number in G0 phase can increase only in the presence of the cells belonging to the G1 phase as a consequence of the transition $\mathrm{G} 1 \rightarrow \mathrm{G} 0$ (promoted by the contact inhibition effect), while the rate of cells entering G0 phase approaches zero when cells complete the transition $\mathrm{G} 1 \rightarrow \mathrm{S}$. As expected, the number of cells in the phase G0 cannot decrease since the transition G1 $\rightarrow \mathrm{G} 0$ has been assumed to be irreversible. Finally, by focusing on the cell number evolution in G1 phase it is apparent that when the rate of transition $\mathrm{G} 1 \rightarrow \mathrm{G} 0$ becomes significant (i.e., after to about $48 \mathrm{~h}$ of cultivation) the cell number belonging to $\mathrm{G} 1$ phase starts to decrease before to experience the transition to the next active phase S. Other important information can be obtained by analyzing the cell population behavior in terms of their volume and age distribution. To this aim, by considering the same data for the simulation illustrated in Fig. 6 a (i.e., $\mathrm{N}_{0}=1.0 \times 10^{5}$ ), the normalized cell distributions on the variables $v$ and $\xi$, at various time, are shown in Fig. 7a and $7 \mathrm{~b}$, respectively.
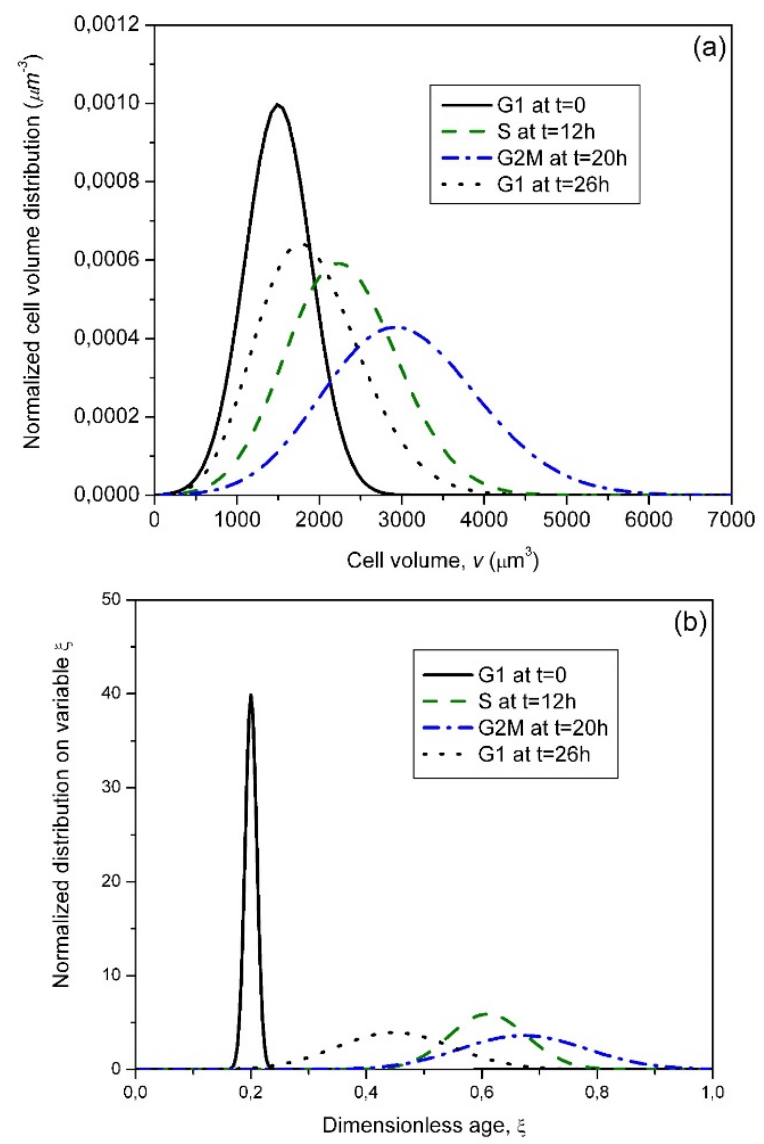

Fig. 7. Normalized distribution on $v$ computed as $\frac{1}{N(t)} \int_{0}^{1} n^{P}(v, \xi, \mathrm{t}) d \xi$ with $\mathrm{P}=\mathrm{G} 1, \mathrm{~S}, \mathrm{G} 2 \mathrm{M}$ (a) and on the variable $\xi$, obtained by $\frac{1}{N(t)} \int_{v_{\min }}^{v_{\max }} n^{P}(v, \xi, \mathrm{t}) d v$ with $\mathrm{P}=\mathrm{G} 1, \mathrm{~S}, \mathrm{G} 2 \mathrm{M}(\mathrm{b})$.

As it can be observed from Fig. 7a, coherently to what shown in Fig. 6a, it is apparent that cells, initially distributed only in $\mathrm{G} 1$ phase, at $\mathrm{t}=12 \mathrm{~h}$ have already experienced the transition $\mathrm{G} 1 \rightarrow \mathrm{S}\left(\mathrm{t}>\tau^{G 1}\right)$. Thus, at this time, cells are in the next $\mathrm{S}$ stage and display a volume distribution with an higher mean value, with respect to the one at $t=0$, since the cell volume at $12 \mathrm{~h}$ is obviously increased. Similarly, at $\mathrm{t}=20 \mathrm{~h}$, after the transition $S \rightarrow G 2 M$, cells belong to G2M phase with a distribution which shows a mean value higher than the one for phase $S$ at $t=12 h$. Finally, for $t=26 h$, cells have already completed their mitotic division and smaller newborn cells are now present in G1 phase displaying a volume distribution with a lower mean value if compared to the one at $t=20 \mathrm{~h}$ for the previous phase (G2M). In terms of age, as shown in Fig. $7 \mathrm{~b}$, when the cultivation time increases it is possible to observe an enlargement of the distribution curve with values spread over the entire range. At $\mathrm{t}=26 \mathrm{~h}$, when the cycle is already completed, the cell population that is back in G1 phase shows an age distribution with a smaller mean value with respect to the one defined at lower cultivation time. Similar results, in terms of normalized distribution on variables $v$ and $\xi$, have been obtained in the case of a seeding with a cell number $\mathrm{N}_{0}=0.5 \times 10^{5} ; 2.0 \times 10^{5} ; 3.0 \times 10^{5}$. These results, for a sake of brevity, are not reported in the present paper. Another important feature of the proposed model is the ability to describe the typical smoothed oscillations in terms of cell count evolution that can occur on the basis of the initial seeding conditions as in the case of cell population initially 
distributed in all active phases described, for example, by the following bivariate normal gaussian function:

$$
\begin{aligned}
n_{0}^{P}(v, \xi)=\frac{N_{0} / 3}{2 \pi \sigma_{v} \sigma_{\xi}} & \exp \left(-\frac{1}{2}\left[\frac{\left(v-\mu_{v}\right)^{2}}{\sigma_{v}^{2}}+\frac{\left(\xi-\mu_{\xi}\right)^{2}}{\sigma_{\xi}^{2}}\right]\right) \text { with P } \\
= & \text { G1 S, G2M }
\end{aligned}
$$

The effect of this particular seeding condition is illustrated in Fig. 8, where the phase cell count, $\mathrm{N}^{\mathrm{P}}$, with $\mathrm{P}=\mathrm{G} 0, \mathrm{G} 1, \mathrm{~S}$, $\mathrm{G} 2 \mathrm{M}$ as well as the total one, $\mathrm{N}$, are reported as a function of time.

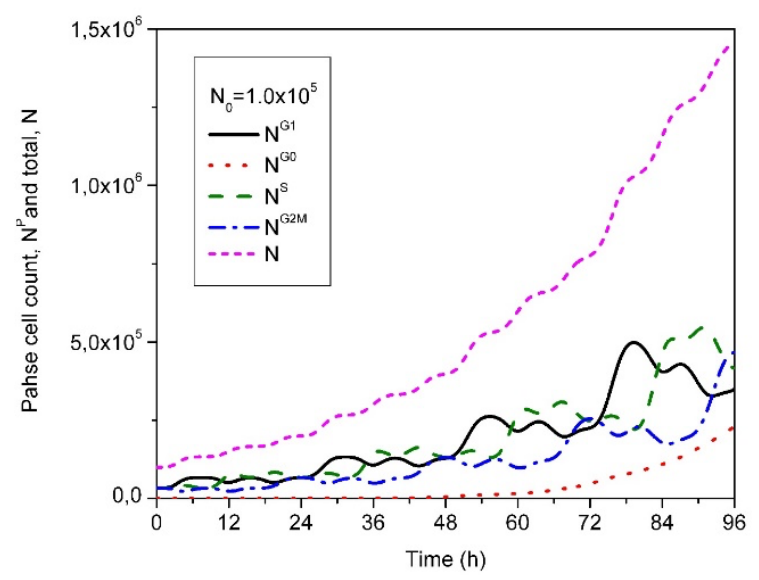

Fig. 8. Phase cell count, $\mathrm{NP}$, with $\mathrm{P}=\mathrm{G}_{1}, \mathrm{G}_{0}, \mathrm{~S}, \mathrm{G} 2 \mathrm{M}$ and total one, $\mathrm{N}$, as a function of time for a seeding with cells in G1, S and G2M phase.

Parameters for this simulation are reported in Table I, where in particular, $\mathrm{N}_{0}=1.0 \times 10^{5}, \mu_{\xi}=0.5$ and $\sigma_{\xi}=0.15$. By comparing the cell count evolution shown in $6 \mathrm{a}$, which refers to a different seeding condition (i.e., cell population initially synchronized in phase G1), with the one of Fig. 8, it is possible to observe, in the latter case, a smoother oscillating behavior. In fact, when cells are initially distributed in all phases, it follows that cells belonging to G2M can divide after few minutes of cultivation, at the same time cells in G1 can enter the next phase $\mathrm{S}$, and cells in $\mathrm{S}$ phase can in turn reach the G2M stage by guaranteeing a sort of continuous behavior in cell expansion. On the other hand, by observing Fig. 8, it is apparent that cell count for each phase fluctuates but with a rising trend during the cultivation.

Finally, let us analyze some possible effect of specific substances added in the culture medium during the cell culture. It is well known that a variety of antagonistic factors may affect the cell cycle progression (Hanahan and Weinberg, 2011). These factors include soluble GFs which promote cell cycle progression, and tumor suppressors, which, on the opposite, inhibit cell proliferation and may cause its arrest (Shapiro and Harper, 1999; Gérard and Goldbeter, 2014). The effect of a GF, which speedups the cell cycle, or of a cytostatic drug which, on the contrary, can cause its arrest or even prevent its starting, can be simulated by suitably changing one parameter of the kinetic model, for example the rate of synthesis of the transcription factor AP1, $v_{S A P 1}$, appearing in (1). It is well known that the presence of GF elicits the activation of signaling pathways leading to the synthesis of AP1 which in turn promotes the synthesis of cyclin D and starts the cell cycle (Gérard and Goldbeter, 2014). Thus, the regulation of AP1 expression level plays a crucial role in the cell cycle machinery. By taking advantage of the biochemical kinetic module of the proposed model it is possible to calculate the variation of the cell cycle lasting as a function of the parameter $v_{S A P 1}$. (cf. Fig. 9).

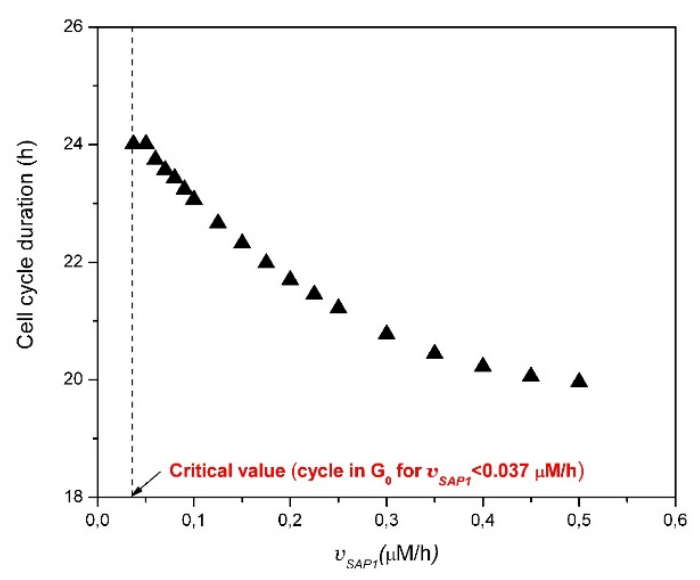

Fig. 9. Variation of the cell cycle duration, calculated by the biochemical kinetic module, as a function of the parameter $v_{S A P 1}$.

All parameters of the biochemical kinetic module used in this simulation are the same of the base case, whose results have been shown in Fig. 4, with the exception of the parameter $v_{S A P 1}$, which was varied from 0.037 to $0.5 \mathrm{mM} / \mathrm{h}$. Correspondingly, the cell cycle duration ranges from 24 to 20 $\mathrm{h}$. Under the critical value of $0.037 \mathrm{mM} / \mathrm{h}$ the cell cycle doesn't start as it may occur when a soluble cytostatic drug is added in the culture medium. On the contrary, the adding specific an external GFs may induce an increase on the rate of synthesis of AP1 leading to a shortening of cell cycle duration as properly simulated by the biochemical kinetic module (Fig. 10), where $v_{S A P 1}$ has been set to $0.5 \mathrm{mM} / \mathrm{h}$.

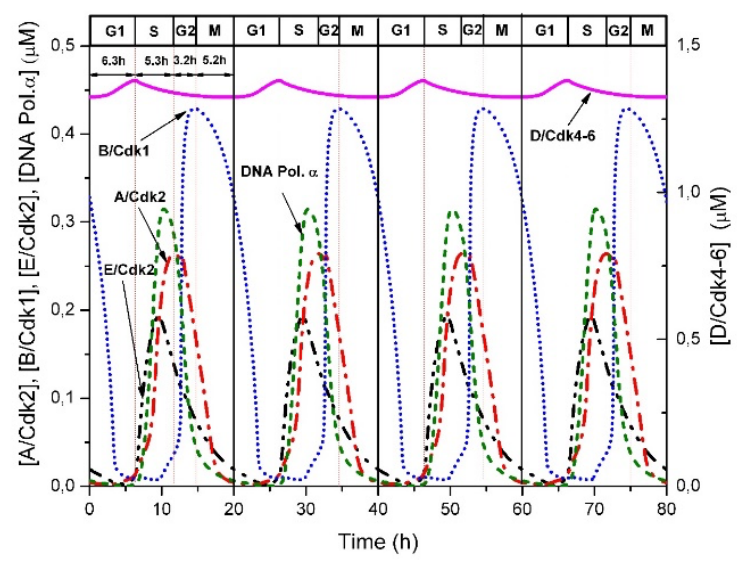

Fig. 10. Expression levels of the main Cyclin-Cdk and of the DNA polymerase $\alpha$ content as a function of time when a soluble GF is added in the culture medium. The duration of a single cell cycle is $20 \mathrm{~h}$.

As it can be observed from Fig. 10 a complete cell cycle is performed within $20 \mathrm{~h}$, being $\tau^{G 1}=6.3 \mathrm{~h}, \tau^{S}=5.3 \mathrm{~h}, \tau^{G 2}=$ $3.2 \mathrm{~h}$ and $\tau^{G 2 M}=5.2 \mathrm{~h}$. In this example, the most relevant effect occurs in the duration of G1 and S phase $\left(\tau^{G 1}=6.3 \mathrm{~h}\right.$ and $\tau^{S}=5.3 \mathrm{~h}$ instead of $\tau^{G 1}=8.4 \mathrm{~h}$ and $\tau^{S}=8.8 \mathrm{~h}$ for the base case whose results are shown in Fig. 4). This effect can be ascribed to the presence of GF that induces a rapid growth of AP1, which in turn leads to a faster increase of cyclin D synthesis, and consequently of its active complex with the kinase Cdk4-6 at the beginning of the cell cycle. On the other hand, there is an early appearance of the complex E/Cdk2 
(after $6.3 \mathrm{~h}$ from the start of the cell cycle when a GF is added instead of $8.4 \mathrm{~h}$ as for the base case) which plays a crucial role in the synthesis phase S. Finally, by taking advantage of the PBM module, the effect of the cycle speeding up induced by an adding of GF during in vitro culture is shown in Fig. 11, where the total cell count is reported as a function of the cultivation time and compared to the corresponding one of the base cases, already depicted in Fig. 5 for $\mathrm{N}_{0}=1.0 \times 10^{5}$.

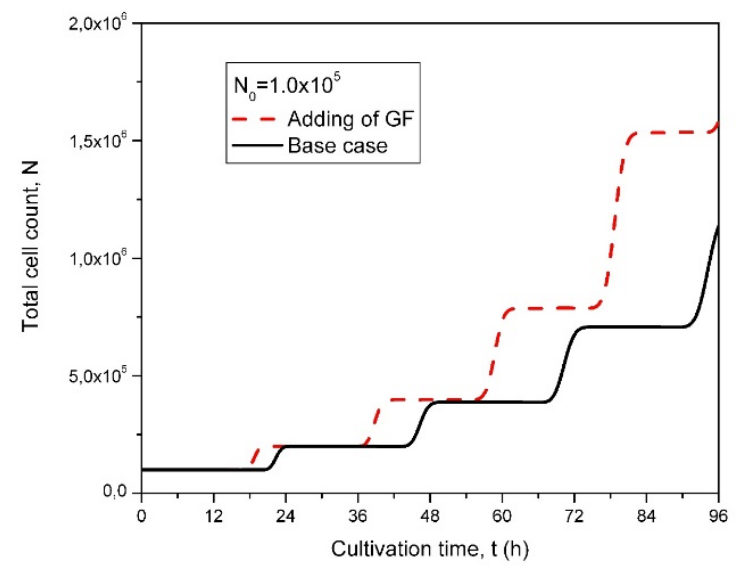

Fig. 11. Total cell count as a function of the cultivation time for the base case (cell cycle completed in $24 \mathrm{~h}$, black solid line) and when GF is added

in the culture medium (cell cycle completed in $20 \mathrm{~h}$, red dashed line).

All parameters used in this simulation, performed by the PBM computational module, are reported in Table I. In particular, in the case of adding GF in the culture medium, the time rates of change on the variable $\xi$ required by PBM, and obtained on the basis of the duration of the phases G1, S, G2M calculated by the biochemical kinetic module, are $r_{\xi}^{G 1}=0.1587=\mathrm{h}^{-1}, r_{\xi}^{S}=0.1887=\mathrm{h}^{-1}, r_{\xi}^{G 2 M}=0.1190=\mathrm{h}^{-}$ ${ }^{1}$. As it might have been expected, simulation results reported in Fig. 11, coherently with the cell cycle timing for the base case (cf. Fig. 4) and when adding GF in culture medium (cf. Fig. 10), clearly show the speeding up due to the extracellular signaling induced by GF in term of the total cell count.

\section{CONCLUDING REMARKS}

A novel mathematical model for simulating the cell cycle progression during in vitro cultivation performed in batch systems is proposed. From the knowledge of the cell phases duration, calculated by means of a detailed biochemical model, the PBM module simulates the time evolution of cells culture in terms of volume, number, and distribution on the phase G0, G1, S and in the lumped one G2M. The proposed model is capable of simulating various situations which may occur during in vitro cultivation as for example those ones arising by the variation of the total number used in seeding or by changing their initial distribution over the cell cycle phases. Results show the ability of the proposed model to interpret the well-known incubation/acclimation time and the typical oscillating behavior during the cell expansion characterized by stationary periods followed by incremental steps. Moreover, the fluctuation in the evolution of the cell number in each phase during the cell culture, which could be experimentally observed through fluorometric methods, is properly simulated by the present model. Other important feature consists in the possibility to interpret the effect of the adding of substances added in the culture medium. A specific example has been discussed regarding the effect of the adding of GF which may reduce the cell cycle duration and consequently can speed up the expansion of the cells during in vitro cultivation.

The results shown represent only a limited portion of the potential of the proposed model which, nevertheless, needs specific experimental validation for demonstrating its full reliability and effective applicability. Work is underway to include for example the effect of cell cycle synchronization factors (Banfalvi, 2017; Pantaleo et al., 2017; Secchi et al., 2020). This aspect is of peculiar importance for infection with lentivirus (Trobridge and Russell, 2004; Bonfalvi, 2017; Pantaleo et al., 2017; Secchi et al., 2020) to generate GM cells, typically for mAb production (or any other recombinant protein).

\section{ACKNOWLEDGEMENT}

This work has been carried out with the financial contribution of the Sardinian Regional Authorities (BIOS Project).

\section{CONFLICT OF INTEREST}

Authors declare that they do not have any conflict of interest.

\section{REFERENCES}

Aguda B.D., Tang,Y. (1999). The kinetic origins of the restriction point in the mammalian cell cycle. Cell Prolif, 32, 321-335.

Ahmadian, M., Tyson, J.J., Peccoud, J., Cao, Y. (2020). A hybrid stochastic model of the budding yeast cell cycle. npj Syst Biol Appl, 6, 1-10.

Almeida S., Chaves, M., Delaunay, F., Feillet, C. (2017). A comprehensive reduced model of the mammalian cell cycle. IFAC-PapersOnLine. 50, $12617-12622$.

Banfalvi, G. (2017) Cell Cycle Synchronization: Methods and Protocols, Methods in Molecular Biology, vol. 1524, $2^{\text {nd }}$ ed., Springer Science+Business Media, Humana Press, New York, USA.

Chen, K.C., Csikasz-Nagy, A., Gyorffy, B., Val, J., Novak, B., Tyson, J.J. (2000). Kinetic analysis of a molecular model of the budding yeast cell cycle. Mol. Biol. Cell, 1, 369-391.

Chen, K.C., Calzone, L., Csikasz-Nagy, A., Cross, F.R., Novak, B., Tyson, J.J. (2004). Integrative analysis of cell cycle control in budding yeast. Mol. Biol. Cell, 15, 3841-3862.

Ciliberto, A., Novak, B., Tyson, J.J. (2003). Mathematical model of the morphogenesis checkpoint in budding yeast. J. Cell Biol, 163, 12431254 .

Csikasz-Nagy, A., Battogtokh, D., Chen, K.C., Novak, B., Tyson J.J. (2006). Analysis of a generic model of eukaryotic cell-cycle regulation. Biophys J, 90, 4361-4379.

Davidich, M.I., Bornholdt, S. (2008). Boolean Network Model Predicts Cell Cycle Sequence of Fission Yeast. PloS One, 3(2), e1672.

Fadda, S., Cincotti, A., Cao G. (2012a). Novel Population Balance Model to Investigate the Kinetics of In Vitro Cell Proliferation: Part I. Model development. Biotechnol. Bioeng. 109, 772-781.

Fadda, S., Cincotti, A., Cao G. (2012b). A Novel Population Balance Model to Investigate Kinetics of In Vitro Cell Proliferation: Part II. Numerical Solution, Parameters' Determination and Model Outcomes. Biotechnol. Bioeng. 109, 782-796.

Florian, J.A., Parker, R.S. (2005). A population balance model of cell cyclespecific tumor growth. IFAC Proceeding Volumes. 38, 72-77.

Fredrickson, A.G., Mantzaris, N.V. (2002). A new set of population balance equations for microbial and cell cultures. Chem. Eng. Sci. 57, 22652278.

Fuentes-Garí, M., Misener, R., García-Munzer, D., Velliou, E., Georgiadis, M.C., Kostoglou, M., Panoskaltsis, E.N., Mantalaris, A. (2015a). A Mathematical Model of Subpopulation Kinetics for the Deconvolution of Leukaemia Heterogeneity. J. R. Soc., Interface, 12, 20150276. 
Fuentes-Garí, M., Misener, R., Georgiadis, M.C., Kostoglou, M., Panoskaltsis, E.N., Mantalaris, A., Pistikopoulos, E.N. (2015b). Selecting a Differential Equation Cell Cycle Model for Simulating Leukemia Treatment. Ind. Eng. Chem. Res. 54, 8847-8859.

Gérard, C., Goldbeter, A. (2009). Temporal self-organization of the cyclin/Cdk network. PNAS, 106, 2164-2168.

Gérard, C., Goldbeter, A. (2012). From quiescence to proliferation: Cdk oscillations drive the mammalian cell cycle. Frontiers in physiology, 3, 413-430.

Gérard, C., Goldbeter, A. (2014). The balance between cell cycle arrest and cell proliferation: control by the extracellular matrix and by contact inhibition, Interface Focus, 4, 1-13.

Hanahan, D., Weinberg, R.A. (2011). Hallmarks of cancer: the next generation. Cell. 144, 646-674.

Hatzis, C., Srienc, F., Fredrickson, A.G. (1995). Multistaged corpuscolar models of microbial growth: Monte Carlo simulations. Biosystems. 36, 19-35.

Karra, S., Sager, B., Karim, M.M. (2010). Multi-scale modeling of heterogeneities in mammalian cell culture processes. Ind. Eng. Chem. Res. 49, 7990-8006.

Liu, Y.H., Bi, J.X., Zeng, A.P., Yuan, J.Q. (2007). A population balance model describing the cell cycle dynamics of myeloma cell cultivation. Biotechnol. Prog. 23, 1198-1209.

Mancuso, L., Liuzzo, M.I., Fadda, S., Pisu, M., Cincotti, A., Arras, M., Desogus, E., Piras, F., Piga, G., La Nasa, G., Concas, A., Cao, G. (2009). Experimental analysis and modeling of in vitro mesenchymal stem cells proliferation. Cell proliferat. 42, 602-616.

Mancuso, L., Liuzzo, M.I., Fadda, S., Pisu, M., Cincotti, A., Arras, M., La Nasa, G., Concas, A., Cao, G. (2010). In vitro ovine articular chondrocyte proliferation: Experiments and modeling. Cell Proliferat. 43, 310-320.

Morgan, D.O. (1995). Principles of Cdk regulation. Nature. 374, 131-134.

Morgan, D.O. (1997). Cyclin-dependent kinases: engines, clocks, and microprocessors. Ann. Rev. Cell Dev. Biol., 13, 261-291.

Morgan, D.O. (2007). The cell cycle: principles of control, New Science Press, London, UK.

Murray, A., Hunt, T. (1993). The Cell Cycle: An Introduction. Oxford University Press, New York, NY, USA.

Nurse, P. (2000). A long twentieth century of cell cycle and beyond. Cell. 100, 71-78.

Pantaleo, A., Kesely, K.R., Pau, M.C., Tsamesidis, I., Schwarzer, E., Skorokhod, O.A., Chien, H.D., Ponzi, M., Bertuccini, L., Low, P.S., Turrini, F.M. (2017). Syk inhibitors interfere with erythrocyte membrane modification during $\mathrm{P}$ falciparum growth and suppress parasite egress. Blood. 130, 1031-1040.

Pisu, M., Concas, A., Cao, G. (2015). A novel quantitative model of cell cycle progression based on cyclin-dependent kinases activity and population balances. Comp. Biol. and Chem, 55,1-13.

Pisu, M., Lai, N., Cincotti, A., Delogu, F., Cao, G. (2003). A simulation model for the growth of engineered cartilage on polymeric scaffolds. Int. J. Chem. React. Eng. http://www.bepress.com/ijcre/vol1/A38.

Pisu, M., Lai, N., Cincotti, A., Concas, A., Cao, G. (2004). Model of engineered cartilage growth in rotating bioreactors. Chem. Eng. Sci. 59, 5035-5040.

Pisu, M., Concas, A., Lai, N., Cao, G. (2006). A novel simulation model for engineered cartilage growth in static systems. Tissue Eng., 12, 2311 2320 .

Pisu, M., Concas, A., Cao, G. (2007). A novel simulation model for stem cells differentiation. J. Biotechnol. 130, 171-182.

Pisu, M., Concas, A., Fadda, S., Cincotti, A., Cao, G. (2008). A simulation model for stem cells differentiation into specialized cells of nonconnective tissues. Computational Biology and Chemistry. 32, 338344.

Qu Z., Weiss, J.N., MacLellan, W.R. (2003). Regulation of the mammalian cell cycle: A model of the G1-to-S transition. Am J Physiol, 284, C349364.

Ramkrishna, D. (2000). Population balances. Theory and applications to particulate systems in engineering. Academic Press Inc., San Diego, USA.

Secchi, C., Orecchioni, M., Carta, M., Galimi, F., Turrini, F., Pantaleo, A. (2020). Signaling Response to Transient Redox Stress in Human Isolated T Cells: Molecular Sensor Role of Syk Kinase and Functional Involvement of IL2 Receptor and L-Selectine. Sensors. 20, 466-482.

Shapiro, G.I., Harper, W. (1999). Anticancer drug targets: cell cycle and checkpoint control. J. Clin. Invest. 104, 645-653.

Sherer, E., Ramkrishna, D. (2008). Stochastic Analysis of Multistate Systems. Ind. Eng. Chem. Res. 47, 3430-3437.

Trobridge, G., Russell, D.W. (2004). Cell Cycle Requirements for Transduction by Foamy Virus Vectors Compared to Those of Oncovirus and Lentivirus Vectors. Journal of virology. 78, 2327-2335.
Tyson, J.J. (1991). Modeling the Cell-Division Cycle - Cdc2 and Cyclin Interactions, Proceedings of the National Academy of Sciences of the United States of America. 88, 7328-7332.

van Vugt, M.A., Bras, A., Medema, R.H. (2004). Polo-like kinase-1 controls recovery from a G2 DNA damage-induced arrest in mammalian cells. Mol Cell. 15, 799-811.

Weis, M.C., Avva, J., Jacobberger, J.W., Sreenath, S.N. (2014). A DataDriven, Mathematical Model of Mammalian Cell Cycle Regulation. PloS One, 9, e97130. 